\title{
World Standard Population
}

National Cancer Institute

\section{Source}

National Cancer Institute. World Standard Population. NCI Thesaurus. Code C71558.

A standard population that covers the whole world. 